\title{
High-extinction-ratio resonant cavity polarizer for quantum-optics measurements
}

\author{
Shailendhar Saraf, ${ }^{1, *}$ Robert L. Byer, ${ }^{2}$ and Peter J. King ${ }^{3}$ \\ ${ }^{1}$ Rochester Institute of Technology, Rochester, New York, USA \\ E. L. Ginzton Laboratory, Stanford University, California, USA \\ ${ }^{3}$ LIGO Project, California Institute of Technology, Pasadena, California, USA \\ *Corresponding author: sxseee@rit.edu
}

Received 8 May 2006; revised 9 March 2007; accepted 6 April 2007;

posted 6 April 2007 (Doc. ID 79395); published 31 May 2007

\begin{abstract}
The use of a high-finesse Fabry-Perot ring cavity with an odd number of reflections as a high-extinctionratio resonant polarizer is shown. Experimental results from quantum-noise measurements using resonant cavities as spatial and spectral filters and precision polarizers are presented. (C) 2007 Optical Society of America

OCIS codes: $130.3120,260.5430,140.3280,270.2500$.
\end{abstract}

Precision optical interferometer experiments like the Laser Interferometer Gravitational Wave Observatory (LIGO) [1] require "quiet" beams that are single frequency, single spatial mode, and single polarization. This typically necessitates the beam from a stable, narrow-linewidth laser like the nonplanar ring oscillator (NPRO) [2] to be spatially and spectrally filtered to reduce the power noise for shot-noise-limited operation. High-finesse Fabry-Perot ring cavities or mode cleaners [3] are useful as spatial and spectral filters for laser beams and have been constructed for LIGO. A polarization-dependent phase shift in a Fabry-Perot ring cavity with an odd number of round-trip reflections enables its use as a resonant polarizer with a high-extinction ratio. A $\pi$-phase-shift differential upon reflection between the two polarizations at angles of incidence less than the polarization angle is the key to the operation of the mode cleaner as a resonant cavity polarizer (RCP). A high-finesse Fabry-Perot ring cavity can be used as a spatial, spectral, and polarization filter integrated into one device. The spatial mode and polarization filtering properties of the $\mathrm{RCP}$ were exploited in an experiment to measure

0003-6935/07/183850-06\$15.00/0

(C) 2007 Optical Society of America the quantum noise in a free-space Nd:YAG saturated amplifier.

The Fresnel coefficients for external reflection from a medium of lower refractive index $\left(n_{i}\right)$ to a higher index $\left(n_{t}\right)$ for $s$ - and $p$-polarization are given by [4]

$$
\begin{aligned}
& r_{s}=-\frac{\sin \left(\theta_{i}-\theta_{t}\right)}{\sin \left(\theta_{i}+\theta_{t}\right)}, \\
& r_{p}=\frac{\tan \left(\theta_{i}-\theta_{t}\right)}{\tan \left(\theta_{i}+\theta_{t}\right)},
\end{aligned}
$$

where $\theta_{i}$ is the angle of incidence at the interface and $\theta_{t}$ is the transmitted angle in the medium with the higher index. $\theta_{i}$ and $\theta_{t}$ are related through Snell's law. At the polarization angle $\theta_{p}$, the reflection coefficient for $p$-polarized, or parallel-polarized, light becomes zero. The polarization angle or the Brewster angle is given by

$$
\theta_{p}=\tan ^{-1}\left(n_{t} / n_{i}\right)
$$

The electric field for $s$-polarization sees a $\pi$-phase shift, while the $p$-polarization sees a $\pi$-phase shift for angles of incidence greater than the polarization (Brewster) angle where the reflected field goes to zero. The $\pi$-phase shift differential between the two 
polarizations at angles less than the polarization angle enables the mode cleaner to be configured as a resonant polarizer. Hence, for an odd number of reflections, the mode cleaner, when locked at resonance for $s$-polarization, is antiresonant for the $p$-polarized light. Similarly, if the mode cleaner is locked at resonance for $p$-polarization, the $s$-polarization field is out of phase with the incident field, and the eigenmode for $s$-polarization is not resonant. Therefore, there are two out-of-phase resonances in the mode cleaner for the two polarizations. These distinct resonances can be expressed in terms of the accumulated phases as

$$
\varphi_{p}-\varphi_{s}=\pi,
$$

where $\varphi_{p}$ and $\varphi_{s}$ are the round-trip phase for $p$ - and $s$-polarized light resonant in the cavity. Therefore, the resonance for $p$-polarization is located half a freespectral range in phase from the contiguous resonance for $s$-polarization. This can be explained in terms of the resonant length of the cavity for an input field at frequency $\omega^{\text {in }}$ with $s$ - and $p$-components at frequencies $\omega_{s}^{\text {in }}$ and $\omega_{p}$ in such that

$$
\begin{aligned}
& L_{p}^{\text {res }}=2 \pi n c / \omega_{p}^{\text {in }}, \\
& L_{s}^{\text {res }}=2 \pi n c / \omega_{s}^{\text {in }}+\pi c / \omega_{s}^{\text {in }}, \\
& \omega_{s}^{\text {in }}=\omega_{p}^{\text {in }}=\omega^{\text {in }},
\end{aligned}
$$

where $L_{s}^{\text {res }}$ and $L_{p}{ }^{\text {res }}$ are the resonant round-trip lengths of the cavity for $s$ - and $p$-polarized light, respectively. The distance offset of the cavity for $s$ - and $p$-polarized light can be written as

$$
L_{s}^{\mathrm{res}}-L_{p}^{\mathrm{res}}=\pi c / \omega^{\mathrm{in}} .
$$

The distance offset can be expressed in terms of the free-spectral range $\Delta L_{\mathrm{FSR}}$ of the cavity as

$$
L_{s}^{\text {res }}-L_{p}{ }^{\text {res }}=\Delta L_{\mathrm{FSR}} / 2 .
$$

Figure 1(a) shows a schematic of the three-mirrorring Fabry-Perot resonator or mode cleaner. Figure 1(b) shows the resonance peaks in transmission for $s$ and $p$-polarized light. Therefore, the extra $\pi$-phase shift results in the resonance for $s$-polarization lying midway between the resonances for $p$-polarization. These two resonances will generally have different values of finesse, because of the difference in the reflectivities of the dielectric-coated mirrors used as the input and output couplers for the cavity. Typical, off-the-shelf dielectric-coated mirrors have a lower reflectivity for $p$-polarized light, and hence the mode cleaners generally have a lower finesse for $p$-polarization. The resonances do not lie exactly midway if the dielectric coating on the mirrors produces a differential phase shift between $s$ - and $p$-polarizations. This can be used to perform precise

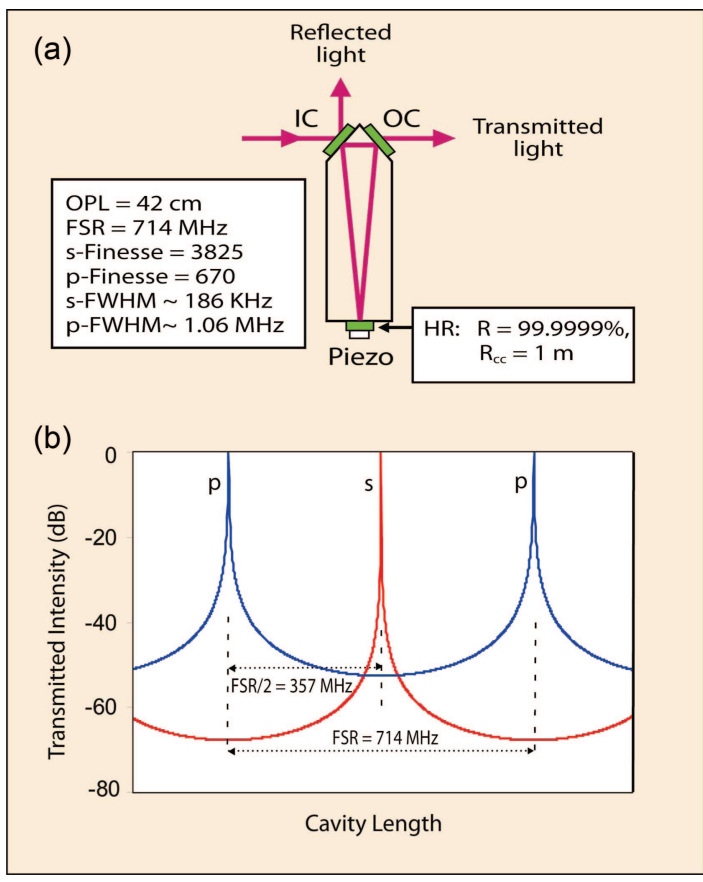

Fig. 1. (Color online) (a) Three-mirror Fabry-Perot cavity referred to as a mode cleaner by LIGO. The cavity is a spatial filter, a spectral filter, and a resonant polarizer integrated into one device. (b) Staggered resonances and calculated transmission for the two polarizations inside the $42 \mathrm{~cm}$ mode cleaner with an odd number of mirrors and an angle of incidence less than the polarization angle at all mirrors. Interferometer length for $p$-polarization resonance falls exactly midway between the lengths for $s$-polarization resonance. The finesse for $p$ - and $s$-polarization is 670 and 3825 , respectively. The extinction for $s$-polarization when the mode cleaner is locked to $p$-polarized light is calculated to be $>52 \mathrm{~dB}$, while the extinction for $p$-polarization when the mode cleaner is locked to $s$-polarized light is calculated to be $>67 \mathrm{~dB}$.

measurements of the relative phase shifts between $s$ - and $p$-polarizations by measuring the offset of the resonance of one polarization referenced to the midway point in the free-spectral range of the other polarization.

These staggered resonances allow the mode cleaner to function as a resonant polarizer with a high extinction ratio. The power transmission through the mode cleaner, locked at resonance, depends on the finesse and the free-spectral range of the cavity and is given by [4]

$$
H\left(v_{\Delta}\right)=\frac{1}{1+\left(\frac{2 F}{\pi}\right)^{2} \sin ^{2}\left(\frac{\pi v_{\Delta}}{\Delta \nu_{\mathrm{FSR}}}\right)},
$$

where $v_{\Delta}$ is the frequency detuning from resonance, $F$ is the finesse of the cavity, and $\Delta \nu_{\mathrm{FSR}}$ is the freespectral range of the cavity in frequency space. As an example, the $42 \mathrm{~cm}$ long mode cleaner shown in Fig. 1(a) has a finesse of 3825 for $s$-polarization and 670 for $p$-polarization. Therefore, using Eq. (7), for the case of the mode cleaner locked at resonance for $s$-polarization, the modeled transmission for $p$ - 


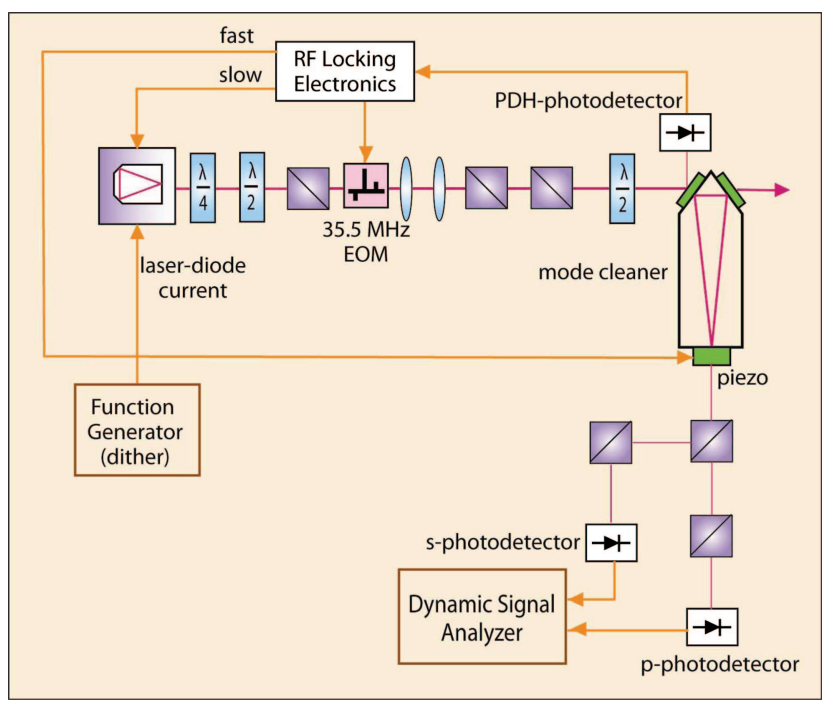

Fig. 2. (Color online) Experimental setup to measure the polarization extinction of a resonant three-mirror cavity. The output of a NPRO is polarized using a pair of precision passive polarizers in order to control the polarization state of the incident light on the mode cleaner to a high degree of fidelity. The extinction ratio of the resonant cavity polarizer is measured using a set of precision polarizers to separate the $s$ - and $p$-components of the leakage light from the back mirror of the mode cleaner. Lock-in techniques are used to measure the polarization state of the cavity output by dithering the laser intensity at a frequency outside the range of the locking servo.

polarized light that resonates half a free-spectral range away is calculated at $-52 \mathrm{~dB}$. However, if the mode cleaner is locked at resonance for $p$-polarization, the calculated transmission for $s$-polarized light is an impressive $-67 \mathrm{~dB}$, as shown in Fig. 1(b). The power of the mode cleaner is now apparent. It is a spatial filter, a spectral filter, and a resonant polarizer integrated into one device.

Figure 2 shows the experimental setup to measure the polarization extinction capabilities of the mode cleaner. An Innolight Mephisto NPRO operating at $1064 \mathrm{~nm}$ was used as the single-frequency, singlespatial-mode laser source for driving the mode cleaner. A quarter-wave, half-wave plate combination was used to convert the elliptical polarized beam from the NPRO into a linearly polarized beam. A New Focus model 4004 broadband electro-optic modulator (EOM) operated at $35.5 \mathrm{MHz}$ was used to impart sidebands for locking the laser to the cavity using the Pound-Drever-Hall (PDH) technique [5]. The beam was linearly polarized using a pair of Karl Lambrecht MGLBAS10 Brewster polarizers for a total extinction of $>90 \mathrm{~dB}$. A half-wave plate was used to adjust the angle of the linearly polarized light to measure the extinction capabilities of the mode cleaner for both polarizations. The mode cleaner was locked to the laser using a combination of fast and slow feedback control in order to have sufficient dynamic range and speed of response of the servo system. The fast servo controls the optical path length of the cavity by driving the piezomounted end mirror of the cavity, while the slow servo adjusts the frequency of the laser through temperature control of the YAG crystal in the NPRO to compensate for slow drifts in the laser frequency. The leakage light from the back mirror of the mode cleaner was analyzed with a set of Karl Lambrecht MGLQD8-V1064 Glan-Laser polarizers with extinction ratios $>60 \mathrm{~dB}$ for a total extinction of $>120 \mathrm{~dB}$ per polarization. The initial experiment was conducted with a mode cleaner that was found to be problematic with a directly transmitted satellite beam in proximity to the transmitted beam from the cavity eigenmode. In order to get around this problem, the detection chain was moved to the back of the mode cleaner. The mode cleaner was subsequently changed with a unit where the satellite beam was scattered out of the cavity, but the detection chain was not moved since the goal of the experiment was a measurement of the polarization extinction ratio. The power in the $s$ - and $p$-polarized light after the polarizers was measured using Thor Labs PDA55 photodetectors. A direct power measurement of the polarization under extinction is difficult since the signal is buried in noise. Therefore, a lock-in technique was used to measure the power in the two polarizations. The laser-diode current of the NPRO was modulated at $10 \mathrm{kHz}$ using a Stanford Research DS435 function generator, resulting in a dither in the laser intensity of $0.02 \%$ RMS. The output of the $s$ - and $p$-photodetectors at the dither frequency was measured with a Stanford Research Dynamic signal analyzer SR785 after amplification by a factor of 200 using a Stanford Research SR560 pre-amp.

The half-wave plate was set up such that the mode cleaner transmitted equal power in the two polarizations. The mode cleaner was first locked to the lowfinesse $p$-resonance, and the outputs of the resonant $p$-photodetector and antiresonant $s$-photodetector were measured at the dither frequency. Next, the mode cleaner was locked to the high-finesse $s$-resonance, and the outputs of the resonant $s$-photodetector and antiresonant $p$-photodetector were measured at the dither frequency. The extinction for the $s$-TEM 00 mode and the $p$-TEM ${ }_{00}$ mode while locked to $p$ polarization and $s$-polarization was measured to be $>59 \mathrm{~dB}$ and $>46 \mathrm{~dB}$, respectively. The measurements were lower than the calculated values due to the imperfect mode matching of the NPRO beam to the $\mathrm{TEM}_{00}$ eigenmode of the cavity. This results in coupling of the transmitted higher-order modes to the antiresonant $\mathrm{TEM}_{00}$ mode for the polarization under extinction.

The best commercially available calcite polarizers have extinction ratios in the range of $60-70 \mathrm{~dB}$. Figure 3 shows the modeled performance of the resonant polarizers for several values of finesse. Lowabsorption substrates and high-reflectivity coatings using ion-beam sputtering techniques on superpolished mirrors are being developed for the LIGO project with absorption losses $<0.5 \mathrm{ppm}$ and reflectivities $>99.9995 \%$ [6]. A three-mirror cavity with the above specifications would have a finesse of $>418878$, a peak cavity transmission of $>0.44$, and a polarization extinction ratio $>108 \mathrm{~dB}$. If the reflec- 


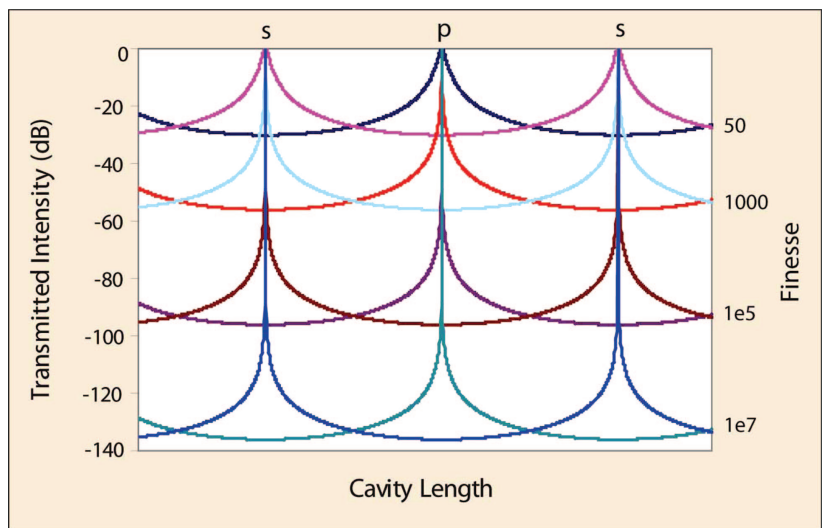

Fig. 3. (Color online) Modeled performance of resonant cavity polarizers with increasing cavity finesse. Using ion-beam sputtered superpolished mirrors with $R>99.9999 \%,>125 \mathrm{~dB}$ polarization extinction ratios can be achieved.

tivity is increased to $99.99995 \%$, for the same absorption losses the finesse would be $>4.2 \times 10^{6}$, a peak cavity transmission of $>0.36$, and a polarization extinction ratio $>128 \mathrm{~dB}$. Advanced LIGO will have a circulating power of about $1 \mathrm{MW}$ in the arm cavities, and at this intracavity power level, a resonant polarizer with a finesse of $10^{7}$ would enable a $100 \mathrm{~mW}$ probe beam to be generated with a $>136 \mathrm{~dB}$ polarization extinction ratio. This remarkable polarization capability along with the spatial and spectral filtering afforded by the high-finesse monolithic cavity would result in beams that are single frequency, single spatial mode, and single polarization with a high degree of pointing stability for sensitive quantumoptics experiments. Additionally, the operating wavelength of the RCP can extend from the UV through the visible to the far IR.

Figure 4(a) shows the experimental setup to measure the quantum noise in a free-space Nd:YAG saturated amplifier using three cavities utilizing the above-mentioned capabilities of resonant cavities. A shot-noise-limited probe beam is generated from a monolithic, single-frequency, single-spatial-mode NPRO. The NPRO is locked to a monolithic, high-finesse and impedance-matched three-mirror Fabry-Perot ring cavity with a finesse of 4400 and 220 and a $3 \mathrm{~dB}$ cutoff frequency of $\sim 87 \mathrm{kHz}$ and $\sim 1.6 \mathrm{MHz}$ for $s$ - and $p$-polarizations, respectively. The NPRO is locked to the monolithic and stable cavity at resonance for $s$-polarization using the PDH technique. The highfinesse mode cleaner reduces the power noise and non-TEM ${ }_{00}$ modes of the NPRO output through temporal and spatial filtering [7]. Additionally, resonant polarizer operation of the mode cleaner described earlier results in $>43 \mathrm{~dB}$ attenuation for light in the "wrong" polarization, which in this instance is $p$-polarization. This results in a $195 \mathrm{~mW}$ probe beam at the output of the mode cleaner prior to the power amplifier that is shot-noise limited at frequencies $>4$ $\mathrm{MHz}$. The high-power beam generated in a masteroscillator power-amplifier chain is also locked to an identical ring Fabry-Perot cavity but with a lower

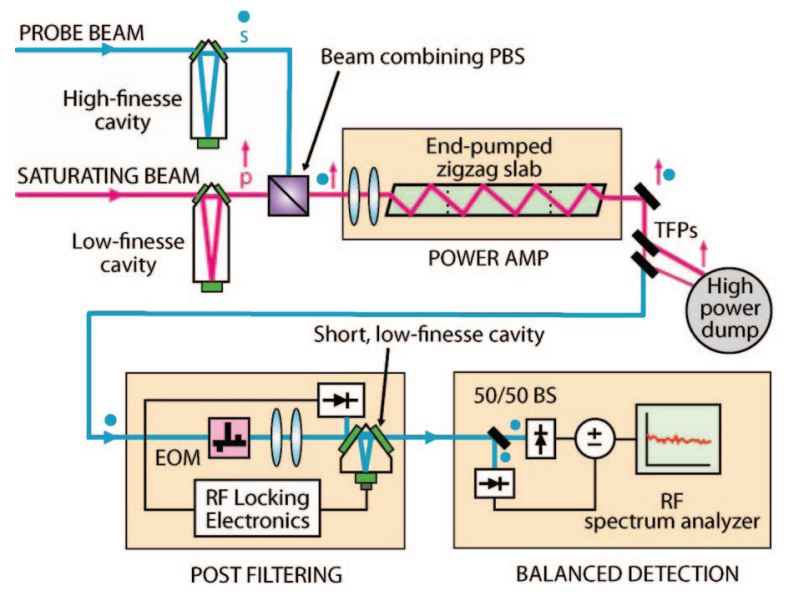

(a)

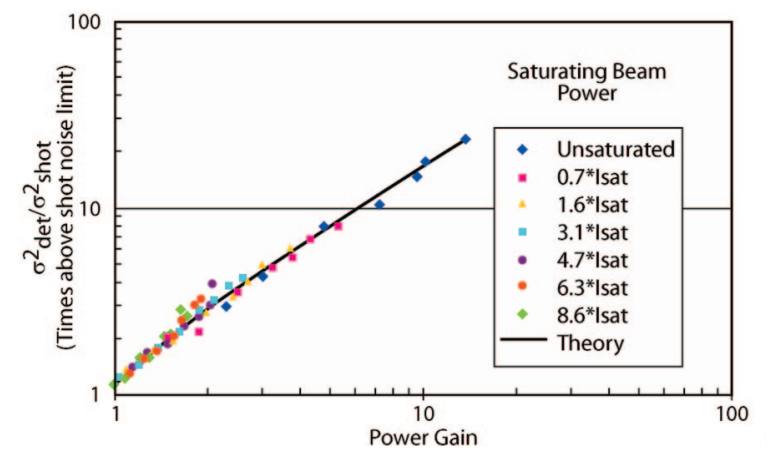

Fig. 4. (a) Layout of the quantum-noise measuring system of a Nd:YAG free-space saturated amplifier. The high-power beam saturates the gain of the $100 \mathrm{~W}$ class slab amplifier and is separated from the precisely overlapped probe beam after the amplifier. The three Fabry-Perot cavities in the setup function as spatial and spectral filters and resonant polarizers. This allows generation of a single-spatial-mode and single-polarization shot-noise-limited probe beam, precision overlap of the high power and probe beam, and finally, precision separation of the beams after amplification. (b) The quantum noise versus power gain plotted for different input intensities of the high-power saturating beam. The theory curve is a plot of the quantum noise Eq. (8).

finesse of approximately 50 and 100 for $p$ - and $s$-polarization, respectively. The high-power beam is locked to the $p$-resonance of the cavity, and again resonant polarizer action results in a $>36 \mathrm{~dB}$ attenuation for $s$-polarized light in the high-power beam. The finesse of the two cavities was set by the reflectivities of the available mirrors and also to avoid mode degradation and mirror damage from thermal effects due to the high intracavity circulating power [8], [9].

The two ring Fabry-Perot cavities have identical constructions except for the reflectivity of the input and output couplers and consequently support identical TEM $_{00}$ eigenmodes. Therefore, the spot sizes of the beams at the output of the cavities are identical and permit the beams to be overlapped with a high degree of precision. The two beams are combined on a polarizing beam splitter equidistant from the two Fabry-Perot cavities. The accurately overlapped high-power beam and the probe beam are then sent through a power amplifier and see the same power 
gain. The two beams are uncorrelated since they are derived from two sources that have no spatial or temporal correlation and additionally have a frequency offset of $750 \mathrm{MHz}$. The frequency offset facilitates separation of the beams after amplification as described later. Since the NPRO and the high-power laser are locked to monolithic and stable cavities, the frequency offset of $750 \mathrm{MHz}$ between the two lasers is stable during the experiment. The power amplifier in the system is an end-pumped $100 \mathrm{~W}$ class Nd:YAG zigzag slab [10]. The slab is pumped at power levels of up to $350 \mathrm{~W}$. The high-power saturating beam is varied from $0 \mathrm{~W}$, which corresponds to a linear unsaturated amplifier, to $27 \mathrm{~W}$, in which case the amplifier is heavily saturated with an input intensity of almost 9 times the saturation intensity for Nd:YAG.

The amplified saturating high-power beam and probe beam are separated after the end-pumped slab amplifier using a pair of thin-film polarizers. The polarizers do not provide rejection of the power coupled from the saturating high-power beam into the probe beam caused by the thermally induced birefringence in the slab at high pump powers [11]. However, further temporal, spatial, and polarization postfiltering is performed by locking the probe beam to the resonance of a short low-finesse mode cleaner operating in $s$-polarization. Figure 5 shows a schematic of

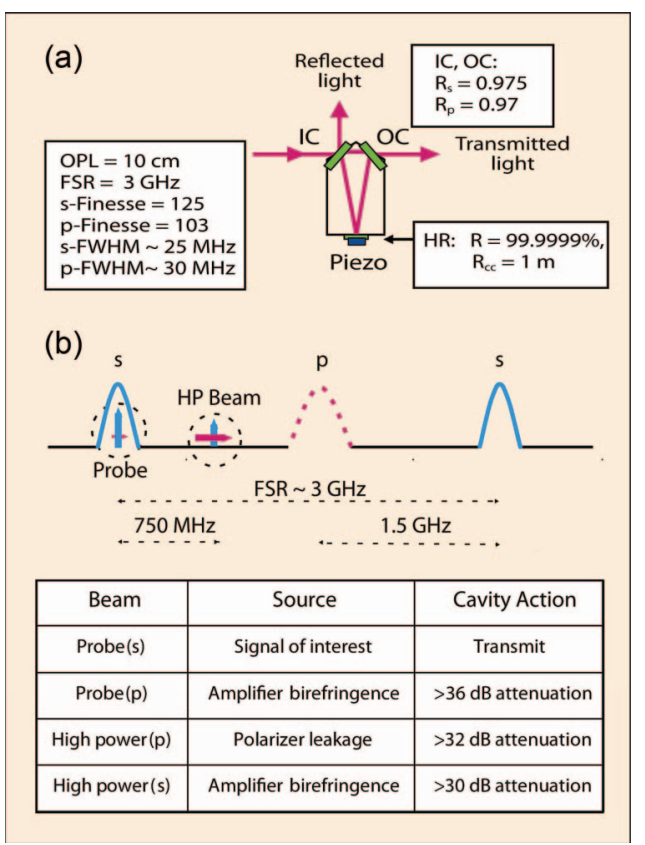

Fig. 5. (Color online) (a) Optical parameters of the short lowfinesse mode cleaner used as a precision filter. The free-spectral range of the cavity is $3 \mathrm{GHz}$, which sets the frequency offset of $750 \mathrm{MHz}$ between the probe and the high-power beam for optimum filtering. (b) Cavity action on the constituent beams at the output of the power amplifier results in transmission of the $s$-polarized probe beam with the added quantum noise due to the amplifier and rejection of other components caused by polarizer leakage and amplifier birefringence. The rejection of the probe in the wrong polarization is due to resonant polarizer action while the rejection of the residual high-power beam is due to resonant polarizer action combined with spectral filtering in the same cavity. the postfiltering Fabry-Perot cavity along with measured cavity filtering action on the constituent beams at the output of the power amplifier. Resonant polarizer action rejects the probe beam in the "wrong" $p$-polarization with an extinction of $>36 \mathrm{~dB}$. The $750 \mathrm{MHz}$ frequency offset between the probe and the high-power beam results in an additional $>30 \mathrm{~dB}$ rejection of the high-power saturating beam in both polarizations. The peak transmission through the postfiltering Fabry-Perot cavity was $>95 \%$. Since attenuation in the path of a noisy beam introduces shot noise, the frequency-dependent attenuation of the cavity along with all attenuations in the path of the probe beam after amplification must be accounted for in the quantum-noise calculations, as described below.

The output of the short low-finesse mode cleaner is then sent though a 50-50 beam splitter for balanced detection to measure the quantum noise vis-à-vis the shot-noise reference level. The narrow passband of the Fabry-Perot cavity along with resonant polarizer operation only allows transmission of the $s$-polarized probe. The cavity completely rejects amplified spontaneous emission, $808 \mathrm{~nm}$ pump light from the amplifier, and coupling of the high-power saturating beam and the probe in the "wrong" polarization, and is the key to the success of the experiment. The probe beam was adjusted at the output of the high-finesse premode cleaner before the amplifier to illuminate the balanced detector with a total photocurrent of $90 \mathrm{~mA}$ for all quantum noise measurements. The noise measurements compensated for the frequencydependent transmission of the postfiltering FabryPerot cavity were averaged over the frequency range of 6.25-15.625 MHz. Figure 4(b) shows the noise measurements in units of "number of times above the shot-noise limit" versus power gain of the probe beam at several saturating power levels. The data points fit excellently over a wide dynamic range of power gain to the quantum-noise equation $[12,13]$

$$
\sigma_{\mathrm{det}}^{2}={\sigma_{\text {shot }}}^{2}\left[1+2 f_{\mathrm{sp}} \eta\left(G_{\mathrm{sat}}-1\right)\right],
$$

where $\sigma_{\operatorname{det}}^{2}$ is the noise power at the detector, $\sigma_{\text {shot }}^{2}$ is the shot-noise power of the amplifier output, $f_{\mathrm{sp}}$ is the spontaneous emission parameter, $\eta$ is the total detection efficiency, and $G_{\text {sat }}$ is the saturated gain of the amplifier. The detection efficiency is the product of the photodetector efficiency, which was measured as 0.82 , and the frequency-dependent attenuation through the postfiltering cavity. The frequency response of the postfiltering cavity for the $s$-polarized probe beam was measured and used to generate the composite frequency-dependent efficiency factor $\eta$ for the theory curve. The spontaneous emission parameter $f_{\mathrm{sp}}$ was calculated at the various power gain settings $G$ using the equation for four-level laser systems,

$$
f_{\mathrm{sp}}=1+\frac{\alpha l}{\ln (G)}
$$




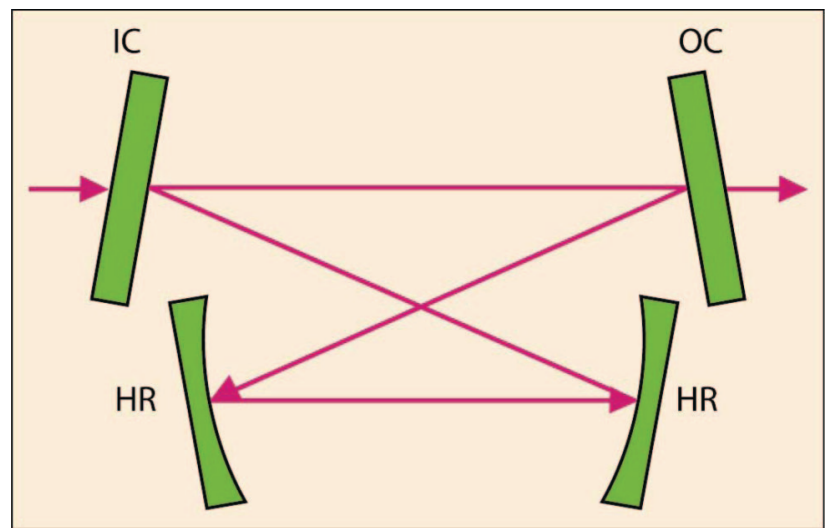

Fig. 6. (Color online) Four-mirror mode cleaner with overlapping $s$ - and $p$-resonances. A tightly focused beam into a Brewster-cut nonlinear crystal like LBO would produce green light for LIGO without astigmatism. The spatial mode does not see any inversion in this configuration.

where $\alpha l$ is the loss traversing the slab measured at $4.1 \%$. The details of the experimental data and Fig. 4(b) are described in Ref. 13.

For $s$-polarized modulating light and $p$-polarized carrier light, the carrier and the modulated sidebands can be transmitted at $\Delta v_{\mathrm{FSR}} / 2$ offset through the same mode cleaner. An alternative is a fourmirror mode cleaner, which has an overlap of $s$ - and $p$-resonances, provided the angle of incidence is below the polarizing angle on none, two, or all four mirrors. The angle of incidence on the two curved mirrors should be small to minimize astigmatism [14]. Figure 6 shows a potential implementation of the mode cleaner. This configuration has the advantage that the spatial mode image is not mirrored. This implementation could be used for second-harmonic generation of the input to yield green output for LIGO.

In summary, we have shown the use of a highfinesse Fabry-Perot cavity or mode cleaner as a highextinction-ratio resonant polarizer. The Fabry-Perot ring cavity with an odd number of reflections below the polarization angle has a net $\pi$-phase shift between the two polarizations, resulting in two distinct and symmetrically spaced resonances in the mode cleaner. Therefore, a mode cleaner locked to resonance of one polarization functions as a spatial and spectral filter and a polarizer integrated in one device. We have demonstrated the use of a Fabry-Perot cavity to generate single-spatial-mode and singlepolarization probe beams and to overlap and separate beams with a high contrast in power levels. The resonances do not lie exactly midway if the dielectric coating on the mirrors produces a differential phase shift between $s$ - and $p$-polarizations. This can be used to perform precise measurements of the relative phase shifts between $s$ - and $p$-polarizations by mea- suring the offset of the resonance of one polarization referenced to the midway point in the free-spectral range of the other polarization.

This work was supported in part by the National Science Foundation under grants PHY-0140297 and PHY-9210038 and in part by the US Army Research Office under ARO grants DAAD19-02-1-0184.

\section{References}

1. B. C. Barish and R. Weiss, "LIGO and the detection of gravitational waves," Phys. Today 52(10), 44-50 (1999).

2. T. J. Kane and R. L. Byer, "Monolithic, unidirectional singlemode Nd:YAG ring laser," Opt. Lett. 10, 65-67 (1985).

3. N. Uehara, E. K. Gustafson, M. M. Fejer, and R. L. Byer, "Modeling of efficient mode-matching and thermal lensing effect on a laser-beam coupling into a mode-cleaner cavity," in Modeling and Simulation of Higher-Power Laser Systems IV, U. O. Farrukhand and S. Basu, eds., Proc. SPIE 2989, 57-69 (1997).

4. E. Hecht, Optics, 4th ed. (Addison-Wesley, 2002).

5. R. W. P. Drever, J. L. Hall, F. V. Kawalski, J. Hough, G. M. Ford, A. J. Munley, and H. Ward, "Laser phase and frequency stabilization using an optical resonator," Appl. Phys. B 31, 97-105 (1983).

6. G. M. Harry, H. Armandula, E. Black, D. R. M. Crooks, G. Cagnoli, M. M. Fejer, J. Hough, S. D. Penn, S. Rowan, R. Route, and P. Sneddon, "Optical coatings for gravitational wave detection," in Advances in Thin Film Coatings for Optical Applications, J. D. T. Kruschwitz and J. B. Oliver, eds., Proc. SPIE 5527, 33-40 (2004).

7. B. Willke, N. Uehara, E. K. Gustafson, R. L. Byer, P. King, S. Seel, and R. L. Savage, Jr., "Spatial and temporal filtering of a $10 \mathrm{~W}$ Nd:YAG laser with a Fabry-Perot ring-cavity premode cleaner," Opt. Lett. 23, 1704-1706 (1998).

8. N. Uehara and K. Ueda, "Accurate measurement of the radius of curvature of a concave mirror and the power dependence in a high-finesse Fabry-Perot interferometer," Appl. Opt. 34, 5611-5619 (1995).

9. L. S. Meng, J. K. Brasseur, and D. K. Neumann, "Damage threshold and surface distortion measurement for highreflectance, low-loss mirrors to $100+\mathrm{MW} / \mathrm{cm}^{2} \mathrm{cw}$ laser intensity," Opt. Express 13, 10085-10091 (2005).

10. G. D. Goodno, S. Palese, J. Harkenrider, and H. Injeyan, "High average-power Yb:YAG end-pumped zig-zag slab laser," in Advanced Solid-State Lasers, C. Marshall, ed., Vol. 50 of OSA Trends in Optics and Photonics Series (Optical Society of America, 2001), pp. 2-4.

11. J. M. Eggleston, T. J. Kane, K. Kuhn, J. Unternahrer, and R. L. Byer, "The slab geometry laser. Part 1. Theory," IEEE J. Quantum Electron. 20, 289-301 (1984).

12. W. M. Tulloch, T. S. Rutherford, E. H. Huntington, R. Ewart, C. C. Harb, B. Willke, E. K. Gustafson, M. M. Fejer, R. L. Byer, S. Rowan, and J. Hough, "Quantum noise in a continuouswave laser-diode-pumped Nd:YAG linear optical amplifier," Opt. Lett. 23, 1852-1854 (1998).

13. S. Saraf, K. Urbanek, R. L. Byer, and P. King, "Quantum noise measurements in a continuous-wave laser-diode-pumped Nd:YAG saturated amplifier,” Opt. Lett. 30, 1195-1197 (2005).

14. H. Kogelnik, E. P. Ippen, A. Dienes, and C. V. Shank, "Astigmatically compensated cavities for CW dye lasers," IEEE J. Quantum Electron. 8, 373-379 (1972). 